OPEN ACCESS

Edited by:

Noemí Sansó,

University of the Balearic Islands,

Spain

Reviewed by:

Kun-Shan Wu,

Tamkang University, Taiwan Jiaying Gong,

The Sixth Affiliated Hospital of Sun

Yat-sen University, China

*Correspondence:

Tao Ba

drbaitao@126.com

${ }^{\dagger}$ These authors have contributed equally to this work and share first authorship

Specialty section:

This article was submitted to

Health Psychology,

a section of the journal

Frontiers in Psychology

Received: 11 November 2021

Accepted: 10 January 2022

Published: 22 February 2022

Citation:

Wang $D$, Chen J, Liu X, Jin Y, Ma $Y$, Xiang $X$, Yang $L$, Song J, Bai $T$ and Hou X (2022) Major Impact of Coping Styles on Anxiety and Depression Symptoms in Healthcare Workers During the Outbreak of COVID-19.

Front. Psychol. 13:813295. doi: 10.3389/fpsyg.2022.813295

\section{Major Impact of Coping Styles on Anxiety and Depression Symptoms in Healthcare Workers During the Outbreak of COVID-19}

\author{
Dongke Wang ${ }^{1+}$, Jie Chen ${ }^{1+}$, Xinghuang Liu', Yan Jin'2, Yanling Ma ${ }^{3}$, Xuelian Xiang', \\ Ling Yang ${ }^{1}$, Jun Song ${ }^{1}$, Tao Bai ${ }^{1 *}$ and Xiaohua Hou ${ }^{1}$
}

'Division of Gastroenterology, Union Hospital, Tongji Medical College, Huazhong University of Science and Technology, Wuhan, China, ${ }^{2}$ Union Hospital, Tongji Medical College, Huazhong University of Science and Technology, Wuhan, China, ${ }^{3}$ Department of Respiratory and Critical Care Medicine, Union Hospital, Tongji Medical College, Huazhong University of Science and Technology, Wuhan, China

Background: In the early days of COVID-19 outbreak, the normally orderly health system was severely challenged by large numbers of feverish patients and shortage of healthcare workers. The outbreak played a harmful role in the mental health of these healthcare workers.

Objective: We aim to assess the prevalence of moderate or severe anxiety and depression symptoms (ADSs) of healthcare workers in different regions during COVID19 disaster and identify the potential risk factors.

Methods: We did a cross-sectional study on ADS of healthcare workers in epicenterHubei province and regions in lower epidemic-other provinces by questionnaire online. The data of ADS, the demographic characteristics, occupational exposure, physical condition, family situation, and coping styles were collected and analyzed.

Results: A total of $24.68 \%$ of the respondents had experienced moderate or severe ADS. Moderate or severe ADSs were in a higher prevalence in Hubei (32.39\%) than other provinces (18.22\%). Suspicious symptoms on their own and in family members were independent risk factors of moderate or severe ADS of all health workers. Working on the frontline was the independent risk factor for participants in Hubei province, whereas quarantine was the independent risk factor for those in other provinces. Moreover, among all participants, those with negative coping style were more than four times more likely to have moderate or severe ADS than those with positive coping style.

Conclusion: Moderate or severe ADSs were in a higher prevalence in healthcare workers of Hubei province during COVID-19 outbreak. The coping style may have major impact on ADS in such situation.

Keywords: COVID-19, healthcare workers, anxiety, depression, coping style 


\section{INTRODUCTION}

On 30 January 2020, given the severity of the outbreak, the World Health Organization (WHO) declared the coronavirus disease 2019 (COVID-19) as a Public Health Emergency of International Concern. By 24 o'clock on 10 February, the cumulative number of confirmed COVID-19 cases in China had reached 42,638, at the same time, Hubei province had 31,728 cumulative confirmed cases of COVID-19 according to the health commission ${ }^{1,2}$. The COVID-19 outbreak was unique in its rapidity of transmission and the large number of healthcare workers who were infected (China-WHO joint investigation report of novel coronavirus pneumonia). The normally orderly health system has been severely challenged by large numbers of feverish patients and shortage of healthcare workers. Great changes had taken place in the work and life of frontline healthcare workers. In addition, many frontline healthcare workers had more than 16 working hours per day on average (Huang and Zhao, 2020). Additionally, we had found that the increased workload was independently related to anxiety, depression, and job burnout among healthcare workers in the previous study (Chen et al., 2020; Liu et al., 2020). Worse still, they were great risk group for infection by the virus. This biodisaster as a life-threatening and life-altering event played a harmful role on the mental health of these healthcare workers.

Coping was defined as thoughts and behaviors that people use to manage the internal and external demands of situations that are appraised as stressful (Folkman and Lazarus, 1980). The earliest classification distinguished coping into problemfocused coping and emotion-focused coping, which were often fitted by later conceptualizations of coping, and also the concepts of positive coping and negative coping. In the majority of studies of coping and adjustment, negative coping has been associated with higher levels of distress (Folkman and Moskowitz, 2004). But it is still unknown whether the coping styles can have an important impact on how healthcare workers respond to this biodisaster and psychological distress. We hypotheses that coping styles and factors related to COVID-19 disaster may potentially contribute to anxiety and depression of healthcare workers in different provinces of China. Therefore, we aim to assess the psychological impact of COVID-19 disaster on Chinese healthcare workers and identify the possible risk factors, especially coping style, associated with anxiety and depression symptoms (ADSs) by a web-based cross-sectional study, to offer favorable conditions in times of extreme distress, such as the current and future pandemics.

\section{MATERIALS AND METHODS}

\section{Study Design and Participants}

From 9 February 2020 to 13 February 2020, we did a crosssectional study of the epidemiology of ADSs of healthcare workers in China. Healthcare workers in hospitals both in epicenter-Hubei province and regions in low epidemic-other provinces who were still at work during the outbreak of COVID-19 were eligible in this survey. Healthcare workers were all local staffs except for support staffs from other regions during this outbreak. The study was conducted by convenience sampling online. The purpose, meaning, and confidentiality of personal information of the research were explained before eligible healthcare workers filled out the selfreported questionnaire. Healthcare workers were recruited when they gave their permission to participate and then voluntarily completed the questionnaire in WeChat small program ${ }^{3}$ after scanning the specific two-dimensional code. Ethics approval was received from the research Ethics Committees of the Wuhan Union Hospital, Tongji Medical College, Huazhong University of Science and Technology.

The demographic characteristics, occupational exposure, physical condition, and family situation were collected in this questionnaire. The generalized anxiety disorder 7-item scale (GAD-7), 9-item Patient Health Questionnaire (PHQ-9), and the 20-item Trait Coping Style Questionnaire (TCSQ) were used to measure psychological status and coping styles (He et al., 2010; Wang et al., 2014; Zhou et al., 2016).

\section{Basic Characteristics}

The demographic characteristics include age, sex (male and female), occupation (doctor, nurse, and other healthcare workers), and title of occupation (primary title, senior title, and none). Primary title included medic, resident doctor, attending doctor, primary nurse, and primary nurse practitioner; senior title included associate chief physician or associate professor, chief physician or professor, nurse-in-charge, deputy chief nurse, and senior nurse. The occupational exposure characteristics included location (Hubei province and other provinces) and working environment (frontline and non-frontline). Hubei province was the epicenter of COVID-19 outbreak, whereas other provinces in China mainland were in lower epidemic. "Frontline" means that healthcare workers directly contacted with patients with COVID-19. Other healthcare workers in hospital were defined as "non-frontline."

Physical condition and family situation were collected by questions answering "yes" or "no." Physical condition was evaluated by "Did you have suspicious symptoms of COVID19 (fever, shortness of breath, cough, sputum, fatigue, muscular soreness, diarrhea, nausea, and vomiting) in last 2 weeks?" Healthcare workers who had one of the suspicious symptoms can choose "yes." The family situation was assessed by the following questions: (1) Are there the old or the child in your family needing to be taken care of? (2) Are you quarantined from your family members? (3) Did your family members have suspicious symptoms in last 2 weeks? Participants who answered "yes" in "question (1)" were defined as family caregivers. Suspicious symptoms in family members were consistent with those in their own physical condition.

\footnotetext{
${ }^{1}$ http://www.nhc.gov.cn

${ }^{2}$ http://wjw.hubei.gov.cn
}

${ }^{3}$ https://web.wechat.com/ 


\section{Measurement of Anxiety and Depression Symptoms}

The degree of ADSs was assessed by GAD-7 and PHQ-9, respectively. There were seven items, each item ranges from 0 to 3 , and the total score of GAD-7 can be divided into four severity levels: severe (>14), moderate (10-14), mild (5-9), and no ( $\leq 4)$ anxiety (Yang et al., 2019). Chinese version of GAD7 was employed in this study, which has good reliability and validity in Chinese population, with Cronbach's alpha coefficient at 0.898 and Kappa value at 0.825 (He et al., 2010; Yang et al., 2019). Additionally, a cutoff score of 10 was recommended for none or mild anxiety and moderate or severe anxiety distinction (He et al., 2010; Kroenke et al., 2010; Tong et al., 2016). There were nine items with each item ranging from 0 to 3. According to the total score, the severity of depression is defined as follows: severe ( $>20)$, moderate to severe (15-20), moderate (10-14), mild (5-9), and no $(\leq 4)$ depression (Wang et al., 2014; Yang et al., 2019). The Chinese version of PHQ9 has also been proved to have high reliability and validity in general Chinese population, whose Cronbach's alpha equals to 0.86 (Wang et al., 2014; Du et al., 2017). Additionally, a total score larger than 9 was recommended as cutoff score to distinguish the none or mild and the moderate or severe depression (Kroenke et al., 2010; Wang et al., 2014; Du et al., 2017). In the analysis, participants who had moderate or severe anxiety or depression symptoms were combined into participants with moderate or severe ADS.

\section{Measurement of Coping Style}

The 20-item TCSQ was employed in this study to evaluate the coping style positive coping (PC) or negative coping (NC) of participants. There are 10 items for each coping style, with each item scores from 1 to 5 . The Chinese version of TCSQ was proved to be valid and reliable in Chinese population, with Cronbach's alpha for each coping style dimension at 0.790 and 0.776, respectively (Ding et al., 2015; Qiao et al., 2016). Individuals are more likely to use the coping style, if its score is higher than that of another coping style (Ding et al., 2015; Zhou et al., 2016).

\section{Statistical Analysis}

Statistical difference in categorical variable was detected by chi-square $\left(\chi^{2}\right)$ test. Student's $t$-test was performed to detect statistical significance in continuous variables. Step-by-step multiple logistic regression was conducted to find independent risk factors for moderate and severe ADS and also calculate odds ratios (ORs) with adjusted for demographic characteristics, such as sex, age, occupation, and so on. The area under the receiver operating characteristic (ROC) curve, also known as $\mathrm{C}$ statistic, was employed to evaluate clinical usefulness of the regression model with a cutoff value of 0.7 (Meurer and Tolles, 2017). We used $\mathrm{R} 3.6 .0^{4}$ to perform all the statistical analyses with a significant double-tailed $p$-value of less than 0.05 .

${ }^{4}$ https://www.r-project.org/

\section{RESULTS}

\section{Basic Characteristics}

Totally, 928 effective questionnaires were recovered online (Table 1). A total of $60.78 \%$ (564/928) of them were doctors, $34.05 \%$ (316/928) were nurses, and 5.17\% (48/928) were other healthcare workers. A total of $45.58 \%$ (423/928) of them came from Hubei Province, which was hardest hit by COVID-19, and 54.42\% (505/928) were from other provinces in China mainland. Almost a quarter of healthcare workers $(24.68 \%$, 229/928) experienced moderate or severe ADS, and 137 of them came from Hubei province. Moderate or severe ADSs were in a higher prevalence $(p<0.001)$ in Hubei $(32.39 \%, 137 / 423)$ than other provinces $(18.22 \%, 92 / 505)$. Coexistence of ADSs was more common $(p<0.001)$ in Hubei province $(15.60 \%, 66 / 423)$ than other provinces $(6.34 \%, 32 / 505)$ (Figure 1). Additionally, the detailed numbers of participants with different anxiety and depression status in Hubei and other provinces are contained in Supplementary Table 1 .

\section{Related Risk Factors of Anxiety and Depression Symptoms Across China}

In the univariate analysis, all of the occupational exposure characteristic, physical condition, family situation, and coping styles had effects on moderate or severe ADSs (Table 1). In the step-by-step logistical analysis, multiple factors can independently influence the occurrence of moderate or severe ADSs (Table 2). The $\mathrm{C}$ statistic of the best regression model of the whole participants in China was 0.752 . Participants with the location of Hubei province (OR 1.600, 95\% CI: 1.1162.294), suspicious symptoms in last 2 weeks (OR 2.254, 95\% CI: $1.612-3.157)$, the suspicious symptoms of family members in last 2 weeks (OR 1.776, 95\% CI: 1.083-2.890), and negative coping style (OR 4.350, 95\% CI: 3.134-6.065) were more likely to have moderate or severe ADS than those without the exposures.

\section{Major Impact of Coping Styles on Anxiety and Depression Symptom}

About $31.25 \%$ (290/928) of healthcare workers had negative coping style and 68.75\% (638/928) had positive coping style. Participants with negative coping style were more than four times more likely to have moderate or severe ADS than those with positive coping style, in both Hubei province and other provinces (Table 3 ). Therefore, negative coping style may have major impact on ADS both in Hubei and other provinces. The detailed prevalence and severity of AD0053 in groups categorized by coping styles and location can be seen in Supplementary Tables 2-4, which were in line with the result of the multiple logistic regressions.

\section{Different Related Risk Factors of Anxiety and Depression Symptom of Hubei Province and Other Provinces}

According to Figure 2, the proportion of the frontline $(p<0.001)$, suspicious symptoms $(p<0.001)$ on their own of 
TABLE 1 | General characteristics of participants according to ADSs $(n=928)$.

\begin{tabular}{|c|c|c|c|c|c|}
\hline Variables & Total & None/mild anxiety and depression (\%) & Moderate/severe anxiety and depression (\%) & Test value $\left(\chi^{2} / t\right)$ & $p$-value \\
\hline \multicolumn{6}{|c|}{ Demographic characteristic } \\
\hline Age & / & $36.50 \pm 8.57$ & $36.59 \pm 8.37$ & -0.135 & 0.893 \\
\hline \multicolumn{4}{|l|}{ Sex } & 1.060 & 0.303 \\
\hline Male & 295 & 229 (77.63) & $66(22.37)$ & & \\
\hline Female & 633 & $470(74.25)$ & $163(25.75)$ & & \\
\hline \multicolumn{4}{|l|}{ Occupation } & 1.019 & 0.601 \\
\hline Doctor & 564 & $422(74.82)$ & $142(25.18)$ & & \\
\hline Nurse & 316 & $243(76.90)$ & $73(23.10)$ & & \\
\hline Others & 48 & $34(70.83)$ & $14(29.17)$ & & \\
\hline \multicolumn{4}{|l|}{ Titles of occupation } & 3.236 & 0.198 \\
\hline Primary title & 552 & $407(73.73)$ & $145(26.27)$ & & \\
\hline Senior title & 328 & $258(78.66)$ & $70(21.34)$ & & \\
\hline None & 48 & $34(70.83)$ & $14(29.17)$ & & \\
\hline \multicolumn{6}{|c|}{ Occupational exposure characteristic } \\
\hline \multicolumn{4}{|l|}{ Location } & 24.109 & $<0.001 * * *$ \\
\hline Hubei province & 423 & $286(67.61)$ & $137(32.39)$ & & \\
\hline Other provinces & 505 & $413(81.78)$ & $92(18.22)$ & & \\
\hline \multicolumn{4}{|l|}{ Working environment } & 15.059 & $<0.001 * * *$ \\
\hline Frontline & 258 & $171(66.28)$ & $87(33.72)$ & & \\
\hline Non-frontline & 670 & $528(78.81)$ & $142(21.19)$ & & \\
\hline \multicolumn{6}{|l|}{ Physical condition } \\
\hline \multicolumn{4}{|c|}{ Having suspicious symptoms related to COVID-19 in last 2 weeks } & 45.516 & $<0.001 * * *$ \\
\hline Yes & 347 & $218(62.82)$ & $129(37.18)$ & & \\
\hline No & 581 & $481(82.79)$ & $100(17.21)$ & & \\
\hline \multicolumn{6}{|l|}{ Family situation } \\
\hline \multicolumn{4}{|c|}{ Being family caregivers of the old or the child } & 4.976 & $0.026 *$ \\
\hline Yes & 585 & $426(72.82)$ & $159(27.18)$ & & \\
\hline No & 343 & $273(79.59)$ & $70(20.41)$ & & \\
\hline \multicolumn{4}{|c|}{ Being quarantined from family } & 14.762 & $<0.001 * * *$ \\
\hline Yes & 212 & $138(65.09)$ & $74(34.91)$ & & \\
\hline No & 716 & $561(78.35)$ & $155(21.65)$ & & \\
\hline \multicolumn{4}{|c|}{ Having suspicious symptoms related to COVID-19 in family members in last 2 weeks } & 16.267 & $<0.001 * * *$ \\
\hline Yes & 95 & $55(57.89)$ & $40(42.11)$ & & \\
\hline No & 833 & $644(77.31)$ & $189(22.69)$ & & \\
\hline \multicolumn{6}{|l|}{ Coping styles } \\
\hline \multicolumn{4}{|c|}{ Coping style assessed by Trait Coping Style Questionnaire } & 93.734 & $<0.001 * * *$ \\
\hline Negative coping style & 290 & $159(54.83)$ & $131(45.17)$ & & \\
\hline Positive coping style & 638 & $540(84.64)$ & $98(15.36)$ & & \\
\hline
\end{tabular}

Values were expressed as $n(\%) .{ }^{*} p<0.05,{ }^{* * *} p<0.001$.

healthcare workers in Hubei province were significantly higher than those in other provinces. On the other hand, negative coping style was more common in Hubei province $(p=0.02)$.

Separate logistic regression analysis was done for different locations. The $\mathrm{C}$ statistic of Hubei province and other provinces was 0.720 and 0.765 , respectively. Among the 11 variables, only three of them working on the frontline (OR 1.704, 95\% CI: 1.0922.679), suspicious symptoms (OR 1.926, 95\% CI: 1.229-3.035) in last 2 weeks and negative coping style (OR 4.309, 95\% CI: 2.760-6.798) were the independent factors for healthcare workers in Hubei province (Table 3). However, in other provinces, suspicious symptoms in last 2 weeks (OR 2.649, 95\% CI: 1.6004.390), quarantine (OR 3.381, 95\% CI: 1.519-7.420), and negative coping style (OR 4.565, 95\% CI: 2.792-7.536) were independent factors (Table 3). Suspicious symptoms in family members in last 2 weeks may be the potential factors to healthcare workers in Hubei and other provinces.

\section{DISCUSSION}

We found that $24.68 \%$ of 928 healthcare workers suffered moderate or severe ADS during the outbreak of COVID-19. Coping style increased the risk of ADS to a great extent. Suspicious symptoms on their own or in family members in last 2 weeks also had independent effect on moderate or severe 


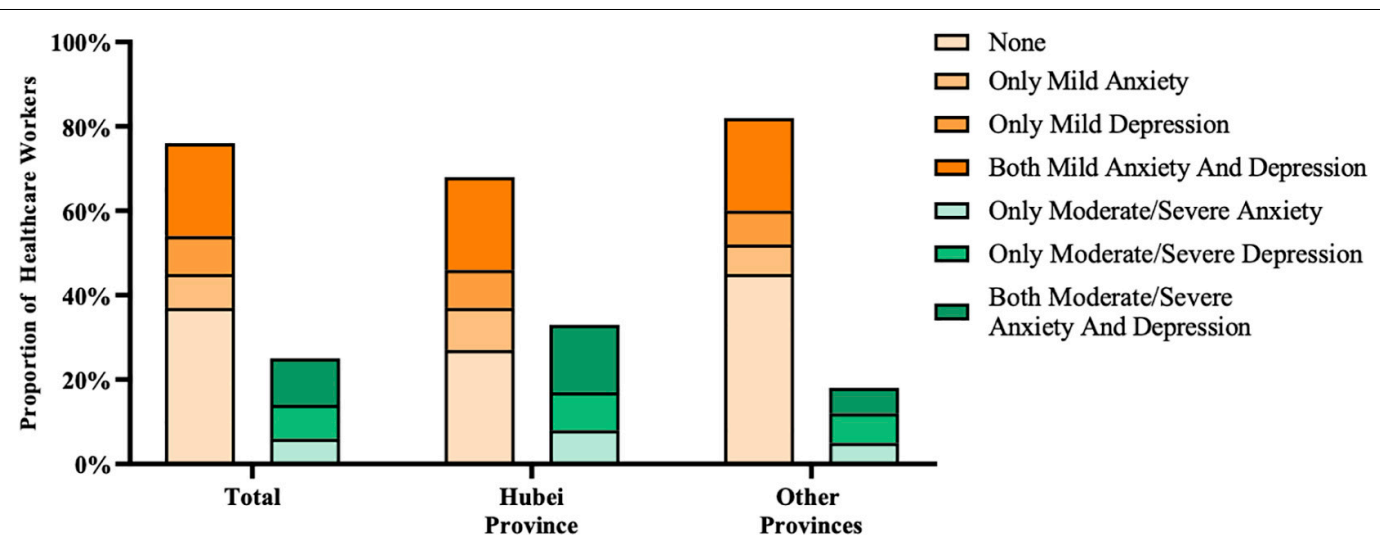

FIGURE 1 | Distribution of ADSs in Hubei province and other provinces. The occurrence of ADSs can be single or coexistent, although most participants in our study had both ADSs. Healthcare workers in Hubei province had a higher prevalence of moderate or severe ADS than those of other provinces.

TABLE 2 | Multiple logistic regression analysis with risk factors for moderate/severe ADSs $(n=928)(C$ statistic $=0.752)$.

\begin{tabular}{|c|c|c|}
\hline Variables & $p$-value & OR $(95 \% \mathrm{Cl})$ \\
\hline \multicolumn{3}{|c|}{ Occupational exposure situation } \\
\hline \multicolumn{3}{|l|}{ Location } \\
\hline Hubei province & $0.011 *$ & $1.600(1.116,2.294)$ \\
\hline Other provinces & & 1 \\
\hline \multicolumn{3}{|l|}{ Work environment } \\
\hline Frontline & 0.062 & $1.431(0.980,2.084)$ \\
\hline Non-frontline & & 1 \\
\hline \multicolumn{3}{|l|}{ Physical condition } \\
\hline \multicolumn{3}{|c|}{ Having suspicious symptoms related to COVID-19 in last 2 weeks } \\
\hline Yes & $<0.001 * * *$ & $2.254(1.612,3.157)$ \\
\hline No & & 1 \\
\hline \multicolumn{3}{|l|}{ Family situation } \\
\hline \multicolumn{3}{|c|}{ Being family caregivers of the old or the child } \\
\hline Yes & 0.080 & $1.370(0.967,1.954)$ \\
\hline No & & 1 \\
\hline \multicolumn{3}{|c|}{ Having suspicious symptoms related to COVID-19 in family members in last 2 weeks } \\
\hline Yes & $0.022 *$ & $1.776(1.083,2.890)$ \\
\hline No & & 1 \\
\hline \multicolumn{3}{|l|}{ Coping style } \\
\hline \multicolumn{3}{|c|}{ Coping style assessed by Trait Coping Style Questionnaire } \\
\hline Negative coping style & $<0.001 * * *$ & $4.350(3.134,6.065)$ \\
\hline Positive coping style & & 1 \\
\hline
\end{tabular}

${ }^{*} p<0.05,{ }^{* * *} p<0.001$.

ADS among all healthcare workers. However, working on the frontline was the independent risk factor for participants in Hubei province, whereas quarantine was an independent risk factor for those in other provinces.

Healthcare workers with both of ADSs were more than those with the single. The two sets of symptoms were different, but researchers have found that many showed a mixed anxiousdepressed symptom picture that cannot be characterized as one type of disorder or the other (Downing, 1974). Thus, the two were perhaps mutually reinforcing each other (Davis et al., 2010). For example, the accumulation of stressful work experiences of COVID-19 plausibly underlined the development of depression following anxiety, and the sad mood induction of depression caused by the spreading and worsening of COVID-19 may sensitize and facilitate the anxiety. Mental disorder screening of healthcare workers should address both anxiety and depression to discover problems.

Hubei province was in a higher prevalence of moderate or severe ADS. Hubei province was the epicenter of the outbreak of COVID-19, and it is where healthcare workers fight the biodisaster first in China. During the outbreak of SARS in 2003-2004, Hong Kong was more seriously hit by SARS than other countries, such as Singapore and Canada. As reported in different studies, mental disorders assessed by General Health 
TABLE 3 | Two separate multiple logistic regression analysis with risk factors for ADSs in Hubei province $(C$ statistic $=0.720)$ and other provinces $(C$ statistic $=0.765)$.

\begin{tabular}{|c|c|c|c|c|}
\hline \multirow[t]{2}{*}{ Variables } & \multicolumn{2}{|c|}{ Hubei province $(n=423)$} & \multicolumn{2}{|c|}{ Other provinces $(n=505)$} \\
\hline & $p$-Value & OR $(95 \% \mathrm{Cl})$ & $p$-Value & OR $(95 \% \mathrm{Cl})$ \\
\hline \multicolumn{5}{|c|}{ Occupational exposure characteristic } \\
\hline \multicolumn{5}{|l|}{ Work environment } \\
\hline Frontline & $0.020^{\star}$ & $1.704(1.092,2.679)$ & :): & $:$ \\
\hline Non-frontline & & 1 & :): & $:$ \\
\hline \multicolumn{5}{|l|}{ Physical condition } \\
\hline \multicolumn{5}{|c|}{ Having suspicious symptoms related to COVID-19 in last 2 weeks } \\
\hline Yes & $0.004^{\star \star}$ & $1.926(1.229,3.035)$ & $<0.001^{\star \star \star}$ & $2.649(1.600,4.390)$ \\
\hline No & & 1 & & 1 \\
\hline \multicolumn{5}{|l|}{ Family situation } \\
\hline \multicolumn{5}{|c|}{ Having suspicious symptoms related to COVID-19 in family members in last 2 weeks } \\
\hline Yes & 0.051 & $1.918(0.995,3.697)$ & 0.073 & $1.963(0.917,4.054)$ \\
\hline No & & 1 & & 1 \\
\hline \multicolumn{5}{|c|}{ Being quarantined from family members } \\
\hline Yes & $:$ & :: & $0.002^{\star \star}$ & $3.381(1.519,7.420)$ \\
\hline No & :) & :) & & 1 \\
\hline \multicolumn{5}{|l|}{ Coping style } \\
\hline \multicolumn{5}{|c|}{ Coping style assessed by Trait Coping Style Questionnaire } \\
\hline Negative coping style & $<0.001^{\star \star \star}$ & $4.309(2.760,6.798)$ & $<0.001^{\star \star \star}$ & $4.565(2.792,7.536)$ \\
\hline Positive coping style & & 1 & & 1 \\
\hline
\end{tabular}

Questionnaire (GHQ) were more common in Hong Kong rather than other countries (Chan and Huak, 2004; Nickell et al., 2004; Tam et al., 2004). But the comparison among these results was lack of rigor, because of the various survey times and the different ways of data collection and analysis. However, the differences in mental symptoms in the epicenter and other regions were compared in our study during the biodisaster. More adverse, mental symptoms in Hubei province can partly be explained by the higher proportion of the frontline, more suspicious symptoms on their own and more negative coping styles. In the current phrase, online psychological assistance specially for healthcare workers, such as remotely delivered psychological therapies, chat lines, and psycho-education, should be provided in time.

Healthcare workers with suspicious symptoms, which were reported as the initial symptoms of SARS-CoV-2 infection (Guan et al., 2020), were more likely to have moderate or severe ADS. An intriguing study suggested that dyspnea one of the usual suspicious symptoms of COVID-19 can reflect an underlying anxiety disorder and there were pathophysiologic relationships between them (Smoller et al., 1996). On the contrary, suspicious symptoms would definitely increase the fear of illness and death, so we think that the scientific research on COVID-19 should be speeded to fight this disease, and necessary protective equipment for healthcare workers should be paid sufficient attention to decrease the incidence of infection.

Suspicious symptoms in family members and family members needing to be taken care of were also independent factors. Uncertainty about the physical health of the family, fear of losing the loved ones, and the decreased family support may contribute to the ADS among participants with poor family situation (Köse et al., 2016). Therefore, the family situation during this biodisaster should not be ignored in the development of ADS. It has been also proved that family caregivers reported increased psychosocial outcomes, so the challenges of caregiving and heavy workload in healthcare workers were exacerbated by the COVID-19 pandemic (Chen et al., 2020; Beach et al., 2021). Increased multifaceted support should be provided during this epidemic, such as facilitating access to telehealth support services for healthcare workers and accessible, affordable, and timely medical care for their family members.

More participants on the frontline (33.72\%) had moderate or severe ADS than the non-frontline $(21.19 \%)$, but the frontline was an independent risk factor in Hubei province not in others. In the epicenter-Hubei province, the workload of healthcare workers may be harder than those in other provinces. During the harder situation, the change in work environment, a lack of professionalism of emerging COVID-19, and the elevated risk of infection of the healthcare workers on the frontline may make them more adverse emotional reactions. Healthcare workers being quarantined from family members had over three times the odds of moderate or severe ADS in other provinces, but it was not the independent factor in Hubei province. The quarantine might be more common in the epicenter than others, so there might be more colleagues standing together and facing all the difficulties. In other provinces, being quarantined was rare, so healthcare workers may be more inundated with the fear of death, the loss of social supports, the shame of SARS-CoV-2 infection, and even feel like discriminated by the society (Im et al., 2018). Therefore, the regional differences should be taken into account when policymaking on mental health of healthcare workers. 


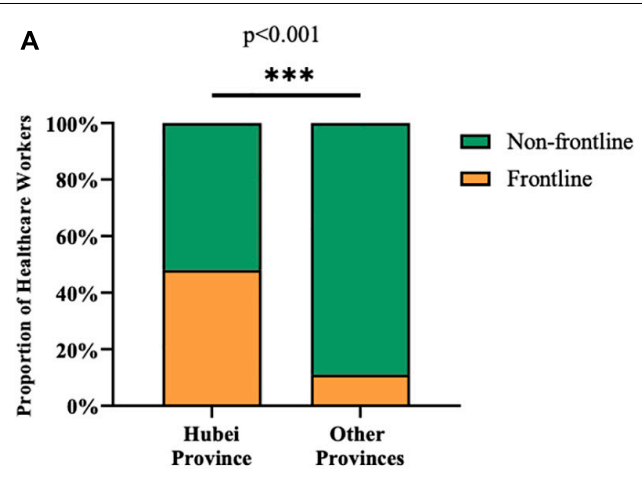

C

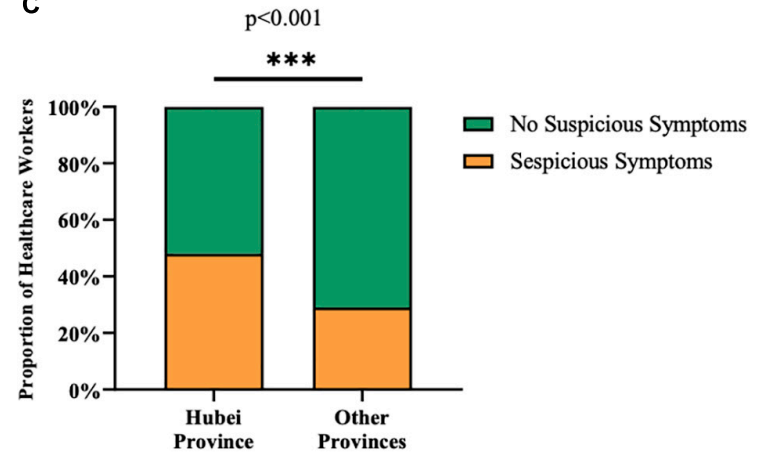

B

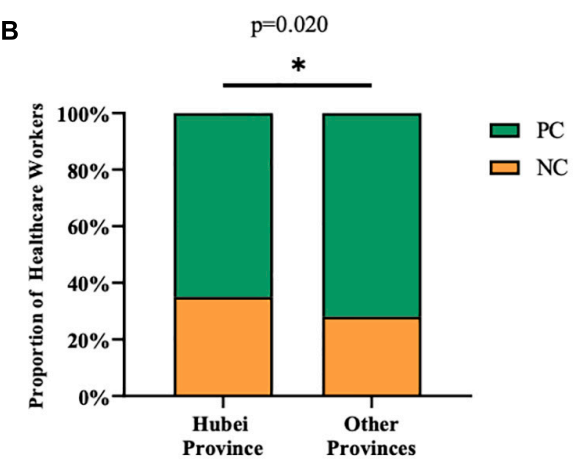

D

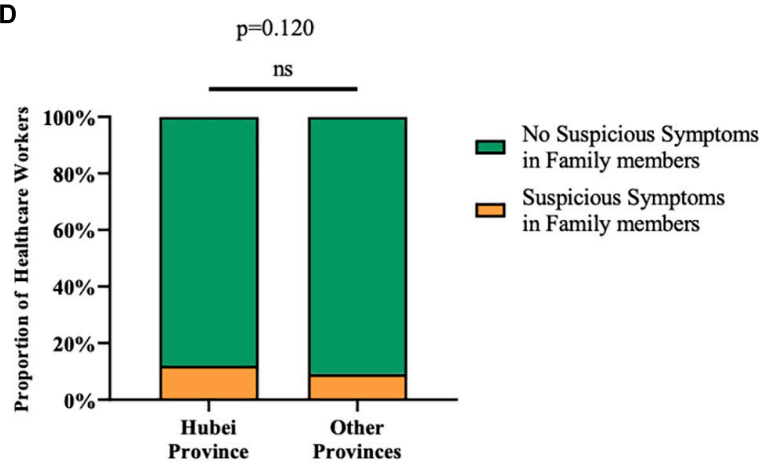

FIGURE 2 | Difference in potential risk factors on ADSs between Hubei province and other provinces. (A) More healthcare workers worked on the frontline in Hubei province (48\%) than those in other provinces (11\%). (B) The proportion of participants with negative coping style was slightly larger in Hubei (35\%) than that of other provinces (28\%). (C) Healthcare workers in Hubei province (48\%) were more frequent to have suspicious symptoms compared with other provinces (29\%). (D) There was no significant difference in the proportion of healthcare workers who had family members with suspicious symptoms between Hubei (12\%) and other provinces (9\%). ${ }^{*} p<0.05,{ }^{* * *} p<0.001,{ }^{n s} p>0.05$

According to the result of multiple logistic regression analysis, coping style had a major impact on the occurrence of moderate or severe ADS both in Hubei and other provinces. A person who applied positive coping may regard stress as opportunities and challenges and then seek solutions to the emerging problems. On the contrary, participants with negative coping are more likely to focus on the negative emotions related to stress event rather than the solution of problem. As demonstrated in previous studies, negative coping would turn to high levels of emotional exhaustion, and positive coping was a positive source to fight against the depressed mood (Ding et al., 2015; Segerstrom and Smith, 2019). Therefore, healthcare workers with negative coping styles referred to negative emotions (such as ADS) when exposing to the stressor of this outbreak. Healthcare administrators should give special attention to coping styles of healthcare workers, and regard them as an important part of promoting the mental health of themselves. Some active interventions have been proved to be effective in the previous studies, including that foster connections of the team, provide education and training to develop active knowledge, skills, attitudes and behaviors, and learn from experiences of other biodisasters (e.g., SARS). Individual strategies and organizational support are both integral to equipping individuals to fight with work-related challenges (Phua et al., 2005; Gillman et al., 2015).
We hope that our findings may also make sense for the future public health crises. Healthcare workers always protect patients from any public health crisis, but the challenges of a public health crisis will inevitably spill over to themselves. At the same time dealing with their own physical health, mental health should also be emphasized. Targeted policy recommendations based on findings from researches could be implemented as soon as possible to avoid serious anxiety or depression disorders of healthcare workers. Besides, there are some limitations within this cross-sectional study. First of all, the only conclusion can be drawn is that those factors discussed above are possible risk factors related to ADS, since the cross-sectional study is not able to explain the relationship between causes and effects. Second, selective bias cannot be avoided when applying convenience sampling by online questionnaires. Even so, the results of our study may provide evidence for further psychological interventions and health policies. The psychological distress of healthcare workers during or post prevalence of COVID-19 still needs deep investigation.

\section{CONCLUSION}

During the outbreak of COVID-19, moderate or severe ADSs were in a higher occurrence rate in healthcare workers in 
the epicenter-Hubei, China. Occupation exposure, physical condition, family situation, and coping style may have independent effects on ADS, but coping style may have a major impact on ADS.

\section{DATA AVAILABILITY STATEMENT}

The raw data supporting the conclusions of this article will be made available by the authors, without undue reservation.

\section{ETHICS STATEMENT}

The studies involving human participants were reviewed and approved by the Research Ethics Committees of Union Hospital, Tongji Medical College, Huazhong University of Science and Technology. Written informed consent for participation was not required for this study in accordance with the National Legislation and Institutional Requirements.

\section{AUTHOR CONTRIBUTIONS}

DW, JC, and XL designed the study. YJ and YM collected the data. LY and XX undertook the statistical analysis. DW and JC wrote the first draft of the manuscript. XL, TB, and JS revised

\section{REFERENCES}

Beach, S. R., Schulz, R., Donovan, H., and Rosland, A. M. (2021). Family caregiving during the COVID-19 pandemic. Gerontologist 61, 650-660. doi: 10.1093/ geront/gnab049

Chan, A. O., and Huak, C. Y. (2004). Psychological impact of the 2003 severe acute respiratory syndrome outbreak on health care workers in a medium size regional general hospital in Singapore. Occup. Med. 54, 190-196. doi: 10.1093/ occmed/kqh027

Chen, J., Liu, X., Wang, D., Jin, Y., He, M., Ma, Y., et al. (2020). Risk factors for depression and anxiety in healthcare workers deployed during the COVID-19 outbreak in China. Soc. Psychiatry Psychiatr. Epidemiol. [Epub ahead of print]. doi: 10.1007/s00127-020-01954-1

Davis, M., Walker, D. L., Miles, L., and Grillon, C. (2010). Phasic vs sustained fear in rats and humans: role of the extended amygdala in fear vs anxiety. Neuropsychopharmacology 35, 105-135. doi: 10.1038/npp.2009.109

Ding, Y., Yang, Y., Yang, X., Zhang, T., Qiu, X., He, X., et al. (2015). The mediating role of coping style in the relationship between psychological capital and burnout among chinese nurses. PLoS One 10:e0122128. doi: 10.1371/journal. pone. 0122128

Downing, R. W. (1974). Mixed anxiety-depression. Fact or myth? Arch. Gen. Psychiatry 30, 312-317. doi: 10.1001/archpsyc.1974.01760090030005

Du, N., Yu, K., Ye, Y., and Chen, S. (2017). Validity study of Patient Health Questionnaire-9 items for Internet screening in depression among Chinese university students. Asia Pac. Psychiatry 9:12266. doi: 10.1111/appy.12266

Folkman, S., and Lazarus, R. S. (1980). An analysis of coping in a middle-aged community sample. J. Health Soc. Behav. 21, 219-239.

Folkman, S., and Moskowitz, J. T. (2004). Coping: pitfalls and promise. Annu. Rev. Psychol. 55, 745-774. doi: 10.1146/annurev.psych.55.090902.141456

Gillman, L., Adams, J., Kovac, R., Kilcullen, A., House, A., and Doyle, C. (2015). Strategies to promote coping and resilience in oncology and palliative care nurses caring for adult patients with malignancy: a comprehensive systematic review. JBI Database Syst. Rev. Implementation Rep. 13, 131-204. doi: 10.11124/ jbisrir-2015- 1898 the manuscript. TB and XH supervised the study. All authors actively participated in the study and reviewed and approved the final manuscript.

\section{FUNDING}

This work was supported by the urgent projects of scientific and technological research on COVID-19 funded by Hubei province (Grant No. 2020FCA014). The funder had no role in the design or conduct of the study; the data collection, management, analysis, or data interpretation; the preparation, review, or approval of the manuscript; or the decision to submit the manuscript for publication.

\section{ACKNOWLEDGMENTS}

We express our gratitude to all the healthcare workers who completed the questionnaire in our study.

\section{SUPPLEMENTARY MATERIAL}

The Supplementary Material for this article can be found online at: https://www.frontiersin.org/articles/10.3389/fpsyg. 2022.813295/full\#supplementary-material

Guan, W. J., Ni, Z. Y., Hu, Y., Liang, W. H., Ou, C. Q., He, J. X., et al. (2020). Clinical characteristics of coronavirus disease 2019 in China. N. Engl. J. Med. 382, 1708-1720. doi: 10.1056/NEJMoa2002032

He, X., Li, C., Qian, J., Cui, H. S., and Wu, W. (2010). Reliability and validity of a generalized anxiety scale in general hospital outpatients. J. Shanghai Arch. Psychiatry 22, 200-203.

Huang, Y., and Zhao, N. (2020). Generalized anxiety disorder, depressive symptoms and sleep quality during COVID-19 outbreak in China: a web-based cross-sectional survey. Psychiatry Res. 288:112954. doi: 10.1016/j.psychres.2020. 112954

Im, S. B., Baumann, S. L., Ahn, M., Kim, H., Youn, B. H., Park, M., et al. (2018). The experience of korean nurses during the middle east respiratory syndrome outbreak. Nurs. Sci. Q. 31, 72-76. doi: 10.1177/0894318417741119

Köse, I., Zincircioððlu, Ç, Öztürk, Y. K., Çakmak, M., Güldoðan, E. A., Demir, H. F., et al. (2016). Factors affecting anxiety and depression symptoms in relatives of intensive care unit patients. J. Intensive Care Med. 31, 611-617. doi: 10.1177/0885066615595791

Kroenke, K., Spitzer, R. L., Williams, J. B., and Löwe, B. (2010). The patient health questionnaire somatic, anxiety, and depressive symptom scales: a systematic review. Gen. Hosp. Psychiatry 32, 345-359. doi: 10.1016/j.genhosppsych.2010. 03.006

Liu, X., Chen, J., Wang, D., Li, X., Wang, E., Jin, Y., et al. (2020). COVID19 outbreak can change the job burnout in health care professionals. Front. Psychiatry 11:563781. doi: 10.3389/fpsyt.2020.563781

Meurer, W. J., and Tolles, J. (2017). Logistic regression diagnostics: understanding how well a model predicts outcomes. JAMA 317, 1068-1069. doi: 10.1001/jama. 2016.20441

Nickell, L. A., Crighton, E. J., Tracy, C. S., Al-Enazy, H., Bolaji, Y., Hanjrah, S., et al. (2004). Psychosocial effects of SARS on hospital staff: survey of a large tertiary care institution. Can. Med. Assoc. J. 170, 793-798. doi: 10.1503/cmaj. 1031077

Phua, D. H., Tang, H. K., and Tham, K. Y. (2005). Coping responses of emergency physicians and nurses to the 2003 severe acute respiratory syndrome outbreak. Acad. Emerg. Med. 12, 322-328. doi: 10.1197/j.aem.2004.11.015 
Qiao, Z., Chen, L., Chen, M., Guan, X., Wang, L., Jiao, Y., et al. (2016). Prevalence and factors associated with occupational burnout among HIV/AIDS healthcare workers in China: a cross-sectional study. BMC Public Health 16:335. doi: 10.1186/s12889-016-2890-7

Segerstrom, S. C., and Smith, G. T. (2019). Personality and coping: individual differences in responses to emotion. Annu. Rev. Psychol. 70, 651-671. doi: 10.1146/annurev-psych-010418-102917

Smoller, J. W., Pollack, M. H., Otto, M. W., Rosenbaum, J. F., and Kradin, R. L. (1996). Panic anxiety, dyspnea, and respiratory disease. Theoretical and clinical considerations. Am. J. Respir. Crit. Care Med. 154, 6-17. doi: 10.1164/ajrccm. 154.1.8680700

Tam, C. W., Pang, E. P., Lam, L. C., and Chiu, H. F. (2004). Severe acute respiratory syndrome (SARS) in Hong Kong in 2003: stress and psychological impact among frontline healthcare workers. Psychol. Med. 34, 1197-1204. doi: 10.1017/ s0033291704002247

Tong, X., An, D., McGonigal, A., Park, S. P., and Zhou, D. (2016). Validation of the Generalized Anxiety Disorder-7 (GAD-7) among Chinese people with epilepsy. Epilepsy Res. 120, 31-36. doi: 10.1016/j.eplepsyres.2015.11.019

Wang, W., Bian, Q., Zhao, Y., Li, X., Wang, W., Du, J., et al. (2014). Reliability and validity of the Chinese version of the patient health questionnaire (PHQ9) in the general population. Gen. Hosp. Psychiatry 36, 539-544. doi: 10.1016/j. genhosppsych.2014.05.021

Yang, Y., Song, Y., Lu, Y., Xu, Y., Liu, L., and Liu, X. (2019). Associations between erectile dysfunction and psychological disorders (depression and anxiety): A cross-sectional study in a Chinese population. Andrologia 51:e13395. doi: 10 1111/and.13395

Zhou, J., Yang, Y., Qiu, X., Yang, X., Pan, H., Ban, B., et al. (2016). Relationship between anxiety and burnout among chinese physicians: a moderated mediation model. PLoS One 11:e0157013. doi: 10.1371/journal.pone.015 7013

Conflict of Interest: The authors declare that the research was conducted in the absence of any commercial or financial relationships that could be construed as a potential conflict of interest.

Publisher's Note: All claims expressed in this article are solely those of the authors and do not necessarily represent those of their affiliated organizations, or those of the publisher, the editors and the reviewers. Any product that may be evaluated in this article, or claim that may be made by its manufacturer, is not guaranteed or endorsed by the publisher.

Copyright (C) 2022 Wang, Chen, Liu, Jin, Ma, Xiang, Yang, Song, Bai and Hou. This is an open-access article distributed under the terms of the Creative Commons Attribution License (CC BY). The use, distribution or reproduction in other forums is permitted, provided the original author(s) and the copyright owner(s) are credited and that the original publication in this journal is cited, in accordance with accepted academic practice. No use, distribution or reproduction is permitted which does not comply with these terms. 